\title{
Sensado de Espectro Local de Banda Ancha para Radios Cognitivos Multi-antena basado en Compleción de Matrices y Muestreo Sub-Nyquist Uniforme en el Dominio Disperso
}

\author{
Evelio Astaiza-Hoyos ${ }^{(1,2)}$, Héctor F. Bermúdez-Orozco(2)* y Luis F. Muñoz ${ }^{(3)}$ \\ (1) GNTT: Grupo de Investigación en Nuevas Tecnologías de Telecomunicaciones. Facultad de Electrónica \\ y Telecomunicaciones, Univ. del Cauca, Popayán, Cauca, Colombia. (e-mail: eastaizah@unicauca.edu.co) \\ (2) GITUQ: Grupo de Investigación en Telecomunicaciones de la Univ. del Quindío. GITUQ, Facultad de \\ Ingeniería, Programa de Ing. Electrónica, Carrera 15 calle 12 Norte, Armenia, Quindío, Colombia. \\ (e-mail: eastaiza@uniquindio.edu.co; hfbermudez@uniquindio.edu.co) \\ (3) LOGICIEL: Grupo de Investigación en Software, Fac. de Ingeniería, Fundación Univ. de Popayán- \\ FUP, Sede Claustro San José calle 5 No. 8-58, Popayán, Cauca, Colombia. \\ (e-mail: Ifredddyms@fup.edu.co)
}

* Autor a quien debe ser dirigida la correspondencia

Recibido Sep. 30, 2016; Aceptado Dic. 1, 2016; Versión final Ene. 10, 2017, Publicado Jun. 2017

\begin{abstract}
Resumen
Este artículo aborda el problema de sensado de espectro local de banda ancha realizado por Radios Cognitivos de múltiples antenas, basado en muestreo sub-Nyquist uniforme en el dominio disperso y compleción de matrices. En el escenario de banda ancha, uno de los mayores problemas para realizar el sensado de espectro es el gran número de muestras a procesar cuando se realiza el muestreo de señales multibanda a tasa iguales o superiores a la tasa de Nyquist. Esto genera grandes tiempos de detección, altos consumos de energía y la necesidad de altas capacidades de procesamiento en los Dispositivos de Radio Cognitivo. Los resultados de simulación demuestran que el método propuesto permite realizar el sensado de espectro de manera eficiente, mejorando el desempeño del sensado.
\end{abstract}

\section{Local Wideband Spectrum Sensing Algorithm for Multiantenna Cognitive Radios based on Matrix Completion and Uniform Sub-Nyquist Sampling in Sparse Domain}

\begin{abstract}
This article addresses the problem of spectrum local broadband sensing by Cognitive Radio of multiple antennas, based on matrix completion and uniform sub-Nyquist sampling in sparse domain. In the wideband environment, one of the biggest problems for spectrum sensing is the large number of samples to be processed when a multiband signal is sampled at or above the Nyquist rate. This generates large detection times, high-energy consumptions and the need for high processing capabilities in Cognitive Radio Devices. The simulation results show that the proposed method allows the efficient sensing of spectrum, improving performance sensing.
\end{abstract}

Keywords: matrix completion; sub-Nyquist sampling; spectrum sensing; cognitive radio 


\section{INTRODUCCION}

El sensado de espectro es universalmente conocido como el principal habilitador de Radio Cognitivo (CR) (Mitola III, 2000), dado que dota al dispositivo de CR de la capacidad de conocimiento del entorno de radio (estado del canal, recursos espectrales disponibles, ocupación del canal, etc.), por lo tanto, permite a los dispositivos cognitivos detectar automáticamente estímulos en su entorno y adaptar de forma inteligente sus parámetros de funcionamiento, garantizando la satisfacción de las necesidades de comunicación del usuario cognitivo (Usuarios Secundarios - SU) utilizando eficientemente y oportunistamente los recursos espectrales no utilizados por los usuarios licenciados (Usuarios Primarios - PU) quienes poseen los derechos de uso del espectro.

Para lograr este propósito, los Dispositivos de Radio Cognitivo (CRD) (Dispositivos asociados a los SU), deben tener la capacidad de identificar con una alta probabilidad la ubicación de los espacios en blanco (WS) (sub-banda ocupada solo por ruido) disponibles en una banda ancha de interés, para ello, se hace necesario implementar en CRD mecanismos de conocimiento de espectro como el sensado de espectro de banda ancha. El sensado de espectro requiere que los CRD realicen de manera permanente la detección de actividad de los PU en el canal de comunicaciones en la banda de interés; por lo que en general, los mecanismos de sensado de espectro se implementan utilizando algoritmos que realizan la digitalización de la señal a tasas iguales o superiores a la tasa de Nyquist, implicando en el escenario de banda ancha el procesamiento de una gran cantidad de muestras, requiriendo de esta manera una alta capacidad de procesamiento de las plataformas que soportarán los algoritmos de sensado de espectro (Mishali et al., 2011).

En los últimos años, basados en la idea de una ocupación dispersa del canal de comunicaciones (i.e. pocas bandas de frecuencia ocupadas y múltiples disponibles), se han trabajado diversidad de propuestas basadas en Sensado Compresivo (CS) (Candes et al.,2006; Baraniuk, 2007), el cual proporciona una manera eficiente de procesar señales dispersas o señales que pueden ser aproximadas adecuadamente por señales dispersas, es decir, que se pueden aproximar por una expansión en términos de una base adecuada, que solo tiene algunos términos significativos, el cual integra muestreo, compresión, reducción de dimensionalidad y optimización, que permitirían realizar el sensado de espectro de banda ancha realizando la digitalización de la señal a tasas inferiores a la tasa de Nyquist. Las técnicas propuestas que permiten realizar un muestreo no uniforme de señales dispersas son: la Conversión Analógico a Información (AIC) (Sun, Nallanathan et al., 2013; Laska et al., 2007), la cual se basa en la idea de realizar un muestreo no uniforme de la señal a la tasa de información, Conversión Modulada de Banda Ancha (Modulated Wideband Conversion - MWC) (H. Sun et al., 2013), se basa en el muestreo generalizado y en la modulación y filtraje de la señal a muestrear sobre múltiples ramas, cada una de ellas, con diferentes funciones moduladoras, lo cual permite explotar las características de las señales multibanda en el dominio frecuencia para realizar un muestreo no uniforme que permita estructurar la señal sub-muestreada en el dominio frecuencia; finalmente, el MultiCoset Sampling (MCS) (H. Sun et al., 2013; Moshe et al., 2009; Yen, et al., 2013), en este, se busca reducir la cantidad de muestras obtenidas de las señales multibanda considerando que el soporte de estas señales en el dominio de la frecuencia es una unión de intervalos finitos.

Así mismo, los sistemas de múltiples antenas son candidatos de implementación en los CRD, y han sido ampliamente utilizados en la actualidad dada su eficacia en múltiples aspectos como incremento de la capacidad y mejor desempeño en canales con desvanecimiento (Goldsmith, 2005). Por lo cual, el uso de este tipo de sistemas en los CRD, es uno de los posibles enfoques para el sensado de espectro, en el cual, se explotan las observaciones realizadas en el dominio espacial. La utilización de estos sistemas de múltiples antenas se ha propuesto en la literatura abierta (Pandharipande y Linnartz, 2007; Wang et al., 2010; Pourgharehkhan et al., 2015; Li et al., 2015). Pandharipande y Linnartz (2007) han demostrado la eficiencia del sensado de espectro en sistemas de múltiples antenas basado en detección de energía (ED) comparado con el caso de única antena. P. Wang et al. (2010) propone una prueba generalizada de máxima verosimilitud para realizar el sensado de espectro en sistemas de múltiples antenas. En este caso la señal del PU se asume como una señal determinista no conocida y evalúa el desempeño del algoritmo propuesto en canal gausiano. En Pourgharehkhan et al. (2015) se estudia el problema de sensado de espectro en sistemas con múltiples antenas asumiendo correlación entre canales de diferentes antenas bajo incertidumbre de calibración del arreglo de antenas, el cual presenta desempeños similares a los obtenidos por sistemas calibrados incluso a SNRs bajas. En Li et al. (2015) se estudia el problema de sensado de espectro en sistemas con múltiples antenas basado en el mayor valor propio de la señal en un escenario en el cual el PU transmite a múltiples valores de potencia, identificando de manera inicial la presencia de PU y luego detectando el nivel de potencia de operación, evaluando su desempeño. En general, los mecanismos de sensado de espectro mencionados anteriormente se implementan utilizando algoritmos que realizan la digitalización de la señal a tasas iguales o superiores a la tasa de Nyquist, por lo tanto, como se indicaba anteriormente, las plataformas hardware que soportarán los algoritmos de sensado requerirán altas capacidades de procesamiento (M. Mishali et al., 2011). 
En este contexto, uno de los principales retos que se enfrentan en CR es la implementación eficiente del sensado de espectro de banda ancha minimizando la tasa de muestreo requerida garantizando alta probabilidad de detección y bajas probabilidades de omisión de detección y falsa alarma, por ello, para realizar la función de sensado de espectro de banda ancha en CR de múltiples antenas, se propone un método novedoso basado en muestreo sub-nyquist uniforme en el dominio disperso, compleción de matrices y en la detección de energía. Lo anterior, basado en la estimación de la matriz de la señal recibida en el dominio disperso, y a partir de las muestras obtenidas de la señal multibanda tomadas con una matriz de muestreo sub-Nyquist uniforme propuesta por los autores, de esta manera, se determina la ocupación o no de los canales que conforman la multibanda sensada, evaluando su eficiencia en función de las probabilidades de detección, falsa alarma y omisión de detección, comparado con algoritmos de sensado de espectro de banda angosta en sistemas de múltiples antenas que operan a la tasa de Nyquist (Pandharipande y Linnartz, 2007; Wang et al., 2010; Pourgharehkhan et al., 2015; Li et al., 2015) y con algoritmos de sensado de espectro de banda ancha en sistemas de única antena basados en CS (Hoyos et al., 2015).

El resto del artículo se encuentra organizado de la siguiente manera: En la sección II se plantea el modelo del Sistema, en la sección III se describe el método propuesto para realizar el sensado de espectro de banda ancha en sistemas de múltiples antenas basado en muestreo sub-Nyquist, en la sección IV se presenta la evaluación del desempeño del algoritmo propuesto contrastando las métricas de evaluación contra las obtenidas del algoritmo de detección de energía secuencial que opera a tasas de muestreo iguales o superiores a la de Nyquist y contra otros métodos de sensado de espectro local basados en sensado compresivo, y finalmente, en la sección $\mathrm{V}$ se presentan las conclusiones del estudio realizado.

\section{MODELO DEL SISTEMA}

Considerando un dispositivo multi-antena de CR que opera sobre una multibanda (licenciada) con un ancho de banda total de $\mathrm{B} \mathrm{Hz}$, el cual se encuentra dividido en $\mathrm{k}$ sub-bandas no traslapadas de igual ancho de banda b, equivalente a $\mathrm{B} / \mathrm{k} \mathrm{Hz}$ por canal como se muestra en la Fig. 1.

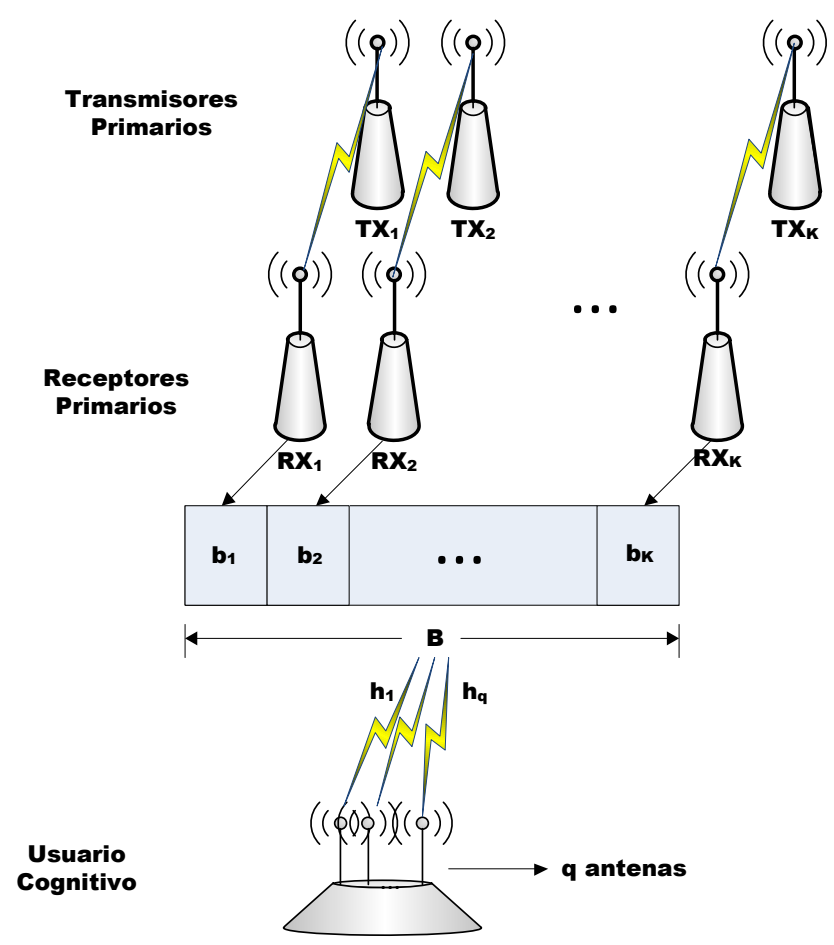

Fig. 1: Escenario Sensado de Espectro de Banda Ancha con Usuario Cognitivo de Múltiples Antenas.

Asumiendo que las muestras de la señal multibanda son variables aleatorias independientes que siguen una distribución normal de media cero y varianza $\sigma_{s}\left(\mathrm{~N}\left(0, \sigma_{s}\right)\right)$, presunción que es válida para cualquier señal multibanda en la cual cada portadora de una sub-banda se modula independientemente por flujos de datos; y que las muestras de ruido en cada antena son variables aleatorias normalmente distribuidas, independientes, de media cero y varianza $\sigma_{n}\left(N\left(0, \sigma_{n}\right)\right)$, la señal recibida en la q-ésima antena puede expresarse como se indica en (1).

$$
X_{i, j}(n)=h_{i, j} s_{i}(n)+W_{i, j}(n)
$$


Donde $\mathrm{X}_{(\mathrm{i}, \mathrm{j})}(\mathrm{n})$ es la $n$-ésima componente de la señal recibida por el SU en la $j$-ésima sub-banda con $j=1,2, . ., \mathrm{k}$ y en la $i$-ésima antena con $i=1,2, \ldots, \mathrm{q}, \mathrm{h}_{(i, j)}$ representa la respuesta del canal en la $j$-ésima sub-banda y en la $i$ ésima antena, $s_{i}(n)$ es la $n$-ésima componente de la señal transmitida por el $j$-ésimo PU sobre la $j$-ésima subbanda y recibida por la $i$-ésima antena del $S U_{\text {y }} \mathrm{W}_{(\mathrm{i}, \mathrm{j}}(\mathrm{n})$ es la $n$-ésima componente del ruido en la $j$-ésima subbanda y en la $i$-ésima antena.

El problema de sensado de espectro en la j-ésima sub-banda puede formularse como un problema de comprobación de hipótesis estadística en el cual se debe escoger entre la hipótesis $H_{(0, j)}$ la cual indica que la $j$-ésima sub-banda se encuentra disponible, y la hipótesis $\mathrm{H}_{(1, j)}$ la cual indica que la $j$-ésima sub-banda se encuentra ocupada, lo anterior puede expresarse de acuerdo a (2).

$$
\left\{\begin{array}{l}
\mathcal{H}_{0, j}: X_{j}=W_{j} \\
\mathcal{H}_{1, j}: X_{j}=h_{j} s_{j}^{*}+W_{j}
\end{array}\right.
$$

donde $X_{j} \in R^{q \times p}$ es la matriz de la señal recibida por el SU en la j-ésima sub-banda, con $q$ igual a la cantidad de antenas en el receptor cognitivo y $p$ igual a la cantidad de muestras tomadas en cada antena por subbanda, $W_{j} \in R^{q \times p}$ es la matriz que representa las componentes de ruido blanco presente en la $j$-ésima subbanda, $h_{j} \in R^{q \times 1}$ es el vector que representa la respuesta del canal en la $j$-ésima sub-banda, finalmente $s_{j}^{*} \in$ $\mathrm{R}^{1 \times p}$ es el vector que representa la señal transmitida por el $j$-ésimo PU sobre la $j$-ésima sub-banda, donde el superíndice * denota transpuesto.

\section{Método Propuesto de Sensado de Espectro de Banda Ancha}

El método de sensado de espectro de banda ancha multi-antena propuesto se ilustra en la fig. 2. Inicialmente la señal multibanda $x(t)$ es recibida por cada una de las antenas del receptor cognitivo, la versión en cada antena se denota por $x_{i}(t)$ con $i=1,2, . ., \mathrm{q}$, posteriormente la señal captada por cada antena se muestrea con el Random Demodulator (RD) (Tropp et al.,2010), donde la operación de muestreo se implementa a través de la matriz de muestreo $A \in R^{m \times n}$, donde $m<n$ con $n$ que representa la cantidad de muestras de la señal cuando se realiza el muestreo a la tasa de Nyquist y $m$ representa la cantidad de muestras Sub-Nyquist tomadas con el RD, obteniendo el vector de muestras $y_{i} \in R^{m}$, donde $y_{i}=A x_{i}$. Posteriormente, el bloque de extracción de características y estimación realiza la estimación de la aproximación dispersa del vector $x_{i}(t)$ que se representa como $x_{i}[n] \in R^{n}$. Finalmente, en el bloque de detección conjunta realiza la detección de WS en la multibanda basado en la energía en cada sub-banda de cada vector de señal estimado por cada antena $x_{i}[n]$ $\epsilon R^{n}$ mediante el criterio de máximo a posteriori, posteriormente se toma la decisión de ocupación final en la multibanda mediante la aplicación de la regla OR entre las decisiones parciales obtenidas en el paso anterior. La figura 2 describe el método.

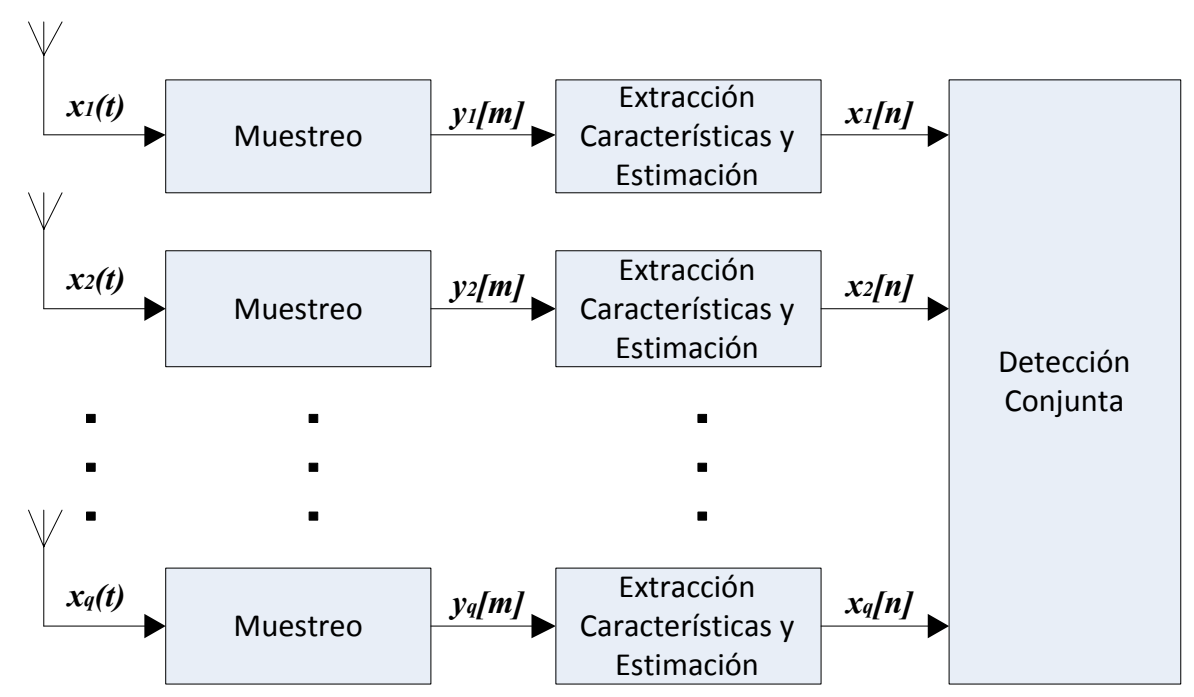

Fig. 2: Escenario Sensado de Espectro de Banda Ancha con Usuario Cognitivo de Múltiples Antenas.

\section{Muestreo}

El muestreo de la señal multibanda $x_{i}(t)$, se realiza mediante la matriz de muestreo $\boldsymbol{A}$ definida como se ilustra en la Fig. 3. 


$$
\left[\begin{array}{cccccccccc}
e^{i \frac{\pi}{10}} & 0 & e^{i \frac{3 \pi}{10}} & 0 & e^{i \frac{5 \pi}{10}} & 0 & e^{i \frac{7 \pi}{10}} & 0 & e^{i \frac{9 \pi}{10}} & 0 \\
0 & 0 & 0 & 0 & 0 & 0 & 0 & 0 & 0 & 0 \\
0 & e^{i \frac{6 \pi}{10}} & 0 & e^{i \frac{12 \pi}{10}} & 0 & e^{i \frac{18 \pi}{10}} & 0 & e^{i \frac{24 \pi}{10}} & 0 & e^{i \frac{30 \pi}{10}} \\
0 & 0 & 0 & 0 & 0 & 0 & 0 & 0 & 0 & 0 \\
e^{i \frac{5 \pi}{10}} & 0 & e^{i \frac{15 \pi}{10}} & 0 & e^{i \frac{25 \pi}{10}} & 0 & e^{i \frac{i 5 \pi}{10}} & 0 & e^{i \frac{45 \pi}{10}} & 0 \\
0 & 0 & 0 & 0 & 0 & 0 & 0 & 0 & 0 & 0 \\
0 & e^{i \frac{14 \pi}{10}} & 0 & e^{i \frac{28 \pi}{10}} & 0 & e^{i \frac{42 \pi}{10}} & 0 & e^{i \frac{56 \pi}{10}} & 0 & e^{i \frac{70 \pi}{10}} \\
0 & 0 & 0 & 0 & 0 & 0 & 0 & 0 & 0 & 0 \\
e^{i \frac{9 \pi}{10}} & 0 & e^{i \frac{27 \pi}{10}} & 0 & e^{i \frac{45 \pi}{10}} & 0 & e^{i \frac{63 \pi}{10}} & 0 & e^{i \frac{81 \pi}{10}} & 0 \\
0 & 0 & 0 & 0 & 0 & 0 & 0 & 0 & 0 & 0
\end{array}\right]
$$

Fig. 3: Matriz de Muestreo Uniforme en el Dominio Disperso Propuesta. Para $n=10$ y Factor de Sub-muestreo 4.

La matriz de la figura 3 cumple con el teorema 1.1 propuesto por Recht (2011). Del proceso de muestreo sub-Nyquist, se obtienen muestras de la forma ilustrada por (3).

$$
y_{i}=A x_{i}
$$

Donde $A$ es la matriz de sensado de tamaño $m \times n, y_{i} \in R^{m}$ es el vector de mediciones y $x_{i} \in R^{n}$ es el vector que representa la señal multibanda $k$-dispersa (Baraniuk, 2007; Hoyos et al., 2015), por lo tanto, las entradas de $y_{i}$ son las muestras sub-Nyquist de $x_{i}$.

\section{Extracción de Características y Estimación}

La característica de la señal que se utiliza para realizar la operación de sensado de espectro es la energía por sub-banda, razón por la cual, el enfoque utilizado se basa en la estimación de la señal dispersa presente en la multibanda sensada. Abordando el problema de encontrar la solución dispersa de $y_{i}=A x_{i}$, utilizando compleción de matrices se debe resolver el problema de optimización planteado en (4)

$$
\min _{x_{i}}\left\|x_{i}\right\|_{*} \text { sujeto a } y_{i, j}=x_{i, j}
$$

Donde $\left\|x_{i}\right\|_{*}$ representa la norma nuclear de $x_{i}, y_{i, j}$ representa la $j$-ésima entrada del vector de muestras del $i$ ésimo CRD y $x_{i, j}$ representa la $j$-ésima entrada del vector de la señal multibanda del $i$-ésimo CRD. Para resolver el problema planteado en (4) se utiliza el algoritmo Singular Value Thresholding (SVT) propuesto en (Cai, Candès, \& Shen, 2010), obteniendo de esta manera la estimación $\hat{x}_{i}$ de la señal presente en el canal a partir de las muestras $y_{i}$.

\section{Detección Conjunta}

La detección conjunta de la ocupación o no de cada sub-banda se realiza en dos etapas: 1.) se decide la ocupación preliminar o no en función de la energía presente en cada sub-banda de la señal estimada en cada antena. 2.) Se toma la decisión conjunta de ocupación final por sub-banda de acuerdo a aplicación de regla OR entre las decisiones preliminares obtenidas para cada antena.

Para realizar la detección de energía para cada sub-banda y para cada antena (etapa 1), se compara la energía de la señal recibida con un umbral de detección, decidiendo de esta forma la ocupación o no de una sub-banda. Por lo tanto la energía presente en cada sub-banda puede calcularse de acuerdo a (5).

$$
\varepsilon_{i, j}(f)=\left|h_{i}\right|^{2} \sum_{S b_{i, j}}\left|\hat{x}_{i}[f]\right|^{2}
$$

Donde $\mathcal{E}_{(i, j)}$ representa la energía en la sub-banda $j$-ésima de la $i$-ésima antena sobre una secuencia de $N$ muestras, $S b_{(i, j)}$ representa la sub-banda $j$-ésima de la $i$-ésima antena, $\boldsymbol{h}_{i}$ representa la respuesta del canal en la $i$-ésima antena y $\widehat{\boldsymbol{X}}_{i}[f]$ representa la señal estimada en el canal en la $i$-ésima antena. Luego, si la energía en la sub-banda $j$-ésima de la $i$-ésima antena es mayor al umbral de decisión $T_{h(i, j)}\left(\mathcal{E}_{i, j}>T_{h(i, j)}\right)$ la decisión tomada es $H_{1, j}$ (sub-banda ocupada) en caso contrario se decide $H_{0, j}$ (sub-banda libre - WS). 
Las probabilidades de detección $P_{d j}$, omisión de detección $P_{m d j}$ y falsa alarma $P_{f j}$ en la sub-banda j-ésima se definen como se indica en (6), (7) y (8).

$$
\begin{aligned}
& P_{d_{j}}=P\left(\mathcal{H}_{1, j} \mid \mathcal{H}_{1, j}\right) \\
& P_{\text {md }_{j}}=P\left(\mathcal{H}_{0, j} \mid \mathcal{H}_{1, j}\right)=1-P_{d j} \\
& P_{f_{j}}=P\left(\mathcal{H}_{1, j} \mid \mathcal{H}_{0, j}\right)
\end{aligned}
$$

Entendiendo como probabilidad de detección a aquella probabilidad de detección correcta de ocupación de una sub-banda o de presencia de señal de un PU en una sub-banda (decidir $H_{1, j}$ cuando $H_{1, j}$ es verdadero), por probabilidad de falsa alarma se entiende la probabilidad asociada a detectar señal de un PU cuando en una sub-banda realmente hay un espacio en blanco (decidir $H_{1, j}$ cuando $H_{0, j}$ es verdadero) y por probabilidad de omisión de detección se entiende aquella probabilidad asociada a detectar un espacio en blanco cuando en una sub-banda hay presencia de señal de un PU (decidir $H_{0, j}$ cuando $H_{1, j}$ es verdadero).

De acuerdo al teorema del límite central, propuesto por Gnedenko y Kolmogorov (1954), si el número de muestras es lo suficientemente grande ( $\geq 10$ en la práctica), las estadísticas de la prueba (media y varianza) de $\mathcal{E}_{i, j}$ asociadas a las hipótesis $H_{0, j}$ y $H_{1, j}$ son normalmente distribuidas asintóticamente y dadas por (9) y (10).

$$
\begin{aligned}
& E\left(\varepsilon_{i, j}\right)= \begin{cases}2 N \sigma_{n_{i, j}}^{2} & : \mathcal{H}_{0, j} \\
\left(S N R_{i, j}+N\right) \sigma_{n, j}^{2} & : \mathcal{H}_{1, j}\end{cases} \\
& \operatorname{Var}\left(\varepsilon_{i, j}\right)= \begin{cases}2 N \sigma_{n_{i, j}}^{4} & : \mathcal{H}_{0, j} \\
2\left(2 S N R_{i, j}+N\right) \sigma_{n, j}^{4} & : \mathcal{H}_{1, j}\end{cases}
\end{aligned}
$$

Con $\left(\sigma_{\mathrm{n} i \mathrm{j}}\right)^{2}$ que denota la energía del ruido en la $i$-ésima antena y $j$-ésima sub-banda y $S N R_{i, j}$ denota la relación señal a ruido en la $i$-ésima antena y $j$-ésima sub-banda.

Luego, las probabilidades de detección y falsa alarma en la $i$-ésima antena y $j$-ésima sub-banda pueden expresarse como se indica en (11) y (12).

$$
\begin{aligned}
& P_{d_{i, j}}=Q\left[\frac{\mathcal{T}_{h_{i, j}}-E\left(\varepsilon_{i, j} \mid \mathcal{H}_{1, j}\right)}{\sqrt[2]{\operatorname{Var}\left(\varepsilon_{i, j} \mid \mathcal{H}_{1, j}\right)}}\right]=Q\left[\frac{\mathcal{T}_{h_{i, j}}-\left(S N R_{i, j}+N\right) \sigma_{n_{i, j}}^{2}}{\sqrt[2]{2\left(2 S N R_{i, j}+N\right) \sigma_{n_{i, j}}^{4}}}\right] \\
& P_{f_{i, j}}=Q\left[\frac{\mathcal{T}_{h_{i, j}}-E\left(\varepsilon_{i, j} \mid \mathcal{H}_{0, j}\right)}{\sqrt[2]{\operatorname{Var}\left(\varepsilon_{i, j} \mid \mathcal{H}_{0, j}\right)}}\right]=Q\left[\frac{\mathcal{T}_{h_{i, j}}-2 N \sigma_{n i, j}^{2}}{\sqrt[2]{2 N \sigma_{n i, j}^{4}}}\right]
\end{aligned}
$$

Donde:

$$
Q(x)=\frac{1}{\sqrt{2 \pi}} \int_{x}^{\infty} e^{-\frac{t^{2}}{2}} d t
$$

Por lo tanto, el umbral de decisión $T_{h i, j}$ para un valor específico de $P_{f i, j}$ está dado por (15).

$$
\mathcal{T}_{h_{i, j}}=Q^{-1}\left(P_{f_{i, j}}\right)^{2} \sqrt{2 N \sigma_{n_{i, j}}^{4}}+2 N \sigma_{n_{i, j}}^{2}
$$

Posteriormente, se procede a decidir de forma conjunta la ocupación por sub-banda mediante regla OR entre las decisiones preliminares en cada antena. De acuerdo a la regla de decisión OR, cuando al menos en una de las $q$ versiones de la sub-banda (una versión por antena) se detecta ocupación, la decisión final es que la sub-banda se encuentra ocupada. Por lo tanto las probabilidades de detección y falsa alarma por sub-banda finales se expresan de acuerdo a (15) y (16). 


$$
\begin{aligned}
& P_{d_{j}}=1-\prod_{i=1}^{q}\left(1-P_{d_{i, j}}\right) \\
& P_{f_{j}}=1-\prod_{i=1}^{q}\left(1-P_{f_{i, j}}\right)
\end{aligned}
$$

Luego, las probabilidades de detección $P_{d j}$, omisión de detección $P_{m d j}$ y falsa alarma $P_{f j}$ en la sub-banda $j$ ésima se definen de acuerdo a (17), (18) y (19).

$$
\begin{aligned}
& P_{d_{j}}=1-\prod_{i=1}^{q}\left(1-Q\left[\frac{\mathcal{T}_{h_{i, j}}-\left(S N R_{i, j}+N\right) \sigma_{n_{i, j}}^{2}}{\sqrt[2]{2\left(2 S N R_{i, j}+N\right) \sigma_{n_{i, j}}^{4}}}\right]\right) \\
& P_{m d_{j}}=\prod_{i=1}^{q}\left(1-Q\left[\frac{\mathcal{T}_{h_{i, j}}-\left(S N R_{i, j}+N\right) \sigma_{n_{i, j}}^{2}}{\sqrt[2]{2\left(2 S N R_{i, j}+N\right) \sigma_{n_{i, j}}^{4}}}\right]\right) \\
& P_{f_{j}}=1-\prod_{i=1}^{q}\left(1-Q\left[\frac{\mathcal{T}_{h_{i, j}}-2 N \sigma_{n_{i, j}}^{2}}{\sqrt[2]{2 N \sigma_{n i, j}^{4}}}\right]\right)
\end{aligned}
$$

Finalmente, se propone calcular las probabilidades de las probabilidades de detección $P_{d}$, omisión de detección $P_{m d}$ y falsa alarma $P_{f}$ de la multibanda de acuerdo a (20), (21) y (22).

$$
\begin{aligned}
& P_{d}=\frac{1}{K} \sum_{j=1}^{K}\left\{1-\prod_{i=1}^{q}\left(1-Q\left[\frac{\mathcal{T}_{h_{i, j}}-\left(S N R_{i, j}+N\right) \sigma_{n i, j}^{2}}{\sqrt[2]{2\left(2 S N R_{i, j}+N\right) \sigma_{n i, j}^{4}}}\right]\right)\right\} \\
& P_{m d}=\frac{1}{K} \sum_{j=1}^{K}\left\{\prod_{i=1}^{q}\left(1-Q\left[\frac{\mathcal{T}_{h_{i, j}}-\left(S N R_{i, j}+N\right) \sigma_{n_{i, j}}^{2}}{\sqrt[2]{2\left(2 S N R_{i, j}+N\right) \sigma_{n i, j}^{4}}}\right]\right)\right\} \\
& P_{f}=\frac{1}{K} \sum_{j=1}^{K}\left\{1-\prod_{i=1}^{q}\left(1-Q\left[\frac{\mathcal{T}_{h_{i, j}}-2 N \sigma_{n i, j}^{2}}{\sqrt[2]{2 N \sigma_{n i, j}^{4}}}\right]\right)\right\}
\end{aligned}
$$

\section{ALGORITMO PROPUESTO}

Para implementar el sensado de espectro, de acuerdo a los procesos descritos de muestreo, extracción de características y estimación y detección conjunta, se propone el algoritmo que se ilustra en la Fig. 4, donde los parámetros de entrada del algoritmo son: la matriz de sensado $A$, la matriz de muestras del canal $Y$, el ancho de banda total de la multibanda $B$, el ancho de banda de cada sub-banda $b$, el tamaño del vector de muestras $m$ y el tamaño del vector señal $n$ (línea 1); el algoritmo propuesto retorna el vector de sub-bandas ocupadas y disponibles en la multibanda ch (línea 2); se utiliza la variable auxiliar Psb para almacenar la potencia por sub-banda de la multibanda (línea 3). El proceso de sensado de espectro inicia calculando el número de sub-bandas en la multibanda (línea 7) y el número de componentes significativas de la multibanda (línea 8), posteriormente, se estima la señal recibida por cada antena mediante la función Estimación_Señal utilizando el algoritmo de reconstrucción de CS propuesto por Natarajan (2006) (línea 10), posteriormente, se calcula la potencia estimada por sub-banda por antena (líneas 13 a 21), finalmente se estima la presencia 0 no de señal en cada sub-banda utilizando la regla OR (líneas 22 a 24). 


\section{Evaluación del Desempeño}

En esta sección se analiza el desempeño del algoritmo propuesto en un escenario en el cual se presenta una señal multibanda compuesta por seis canales de $3.3 \mathrm{MHz}$ cada uno, los cuales aleatoriamente presentan ocupación, el objetivo es evaluar las condiciones bajo las cuales el algoritmo presenta un desempeño adecuado para el sensado de espectro, en comparación con el desempeño que presenta el algoritmo de detección secuencial de energía que funciona a tasas de muestreo iguales o superiores a la tasa de Nyquist.

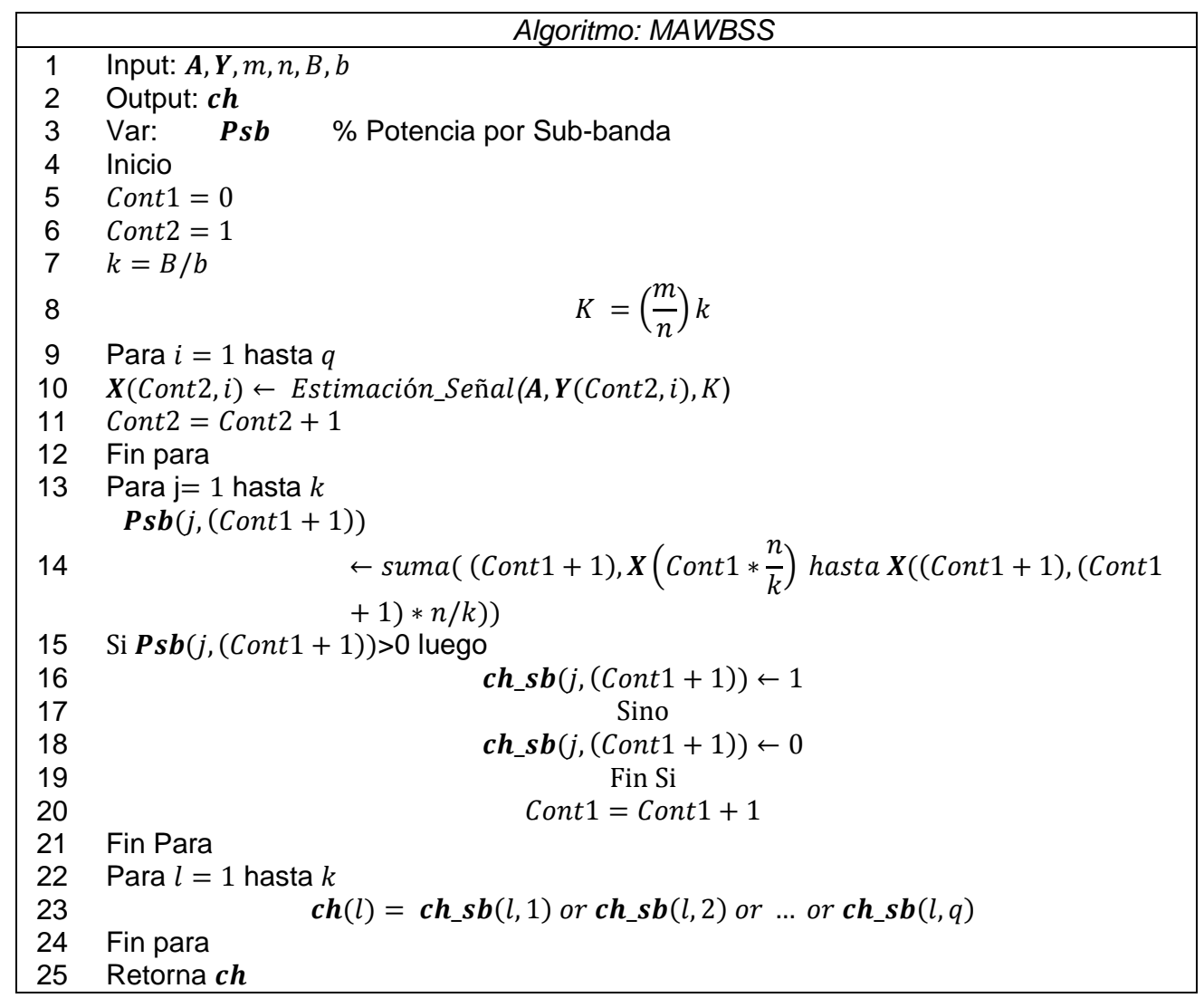

Fig. 4: Algoritmo de Sensado de Espectro de Banda Ancha MAWBSS.

\section{Escenario y Parámetros de Simulación}

En la implementación de la simulación del algoritmo de sensado de espectro de banda ancha propuesto, se genera una señal multibanda de acuerdo con los parámetros de simulación que se muestran en la Tabla 1.

\section{RESULTADOS Y DISCUSIÓN}

Para evaluar el desempeño del algoritmo de sensado de espectro propuesto, se utilizan como métricas la probabilidad de falsa alarma, la probabilidad de omisión de detección y la probabilidad de detección; analizadas en función de la cantidad de componentes recuperadas en la señal estimada mediante el algoritmo de reconstrucción propuesto y en función de la relación señal a ruido de la multibanda generada, comparadas con las métricas obtenidas de los algoritmos propuestos en Pandharipande y Linnartz (2007), P. Wang et al. (2010), P. Wang et al. (2015), Li et al. (2015), Olabiyi y Annamalai (2012a, 2012b), Sun et al. (2015), Tian (2008), Haque et al. (2015) y Wang et al. (2015); los resultados obtenidos se muestran en las Fig. 5 y 6.

En la Fig. 5 se observa el desempeño del algoritmo propuesto contra el desempeño del detector de energía secuencial basado en muestreo Nyquist propuestos por Olabiyi y Annamalai (2012a, 2012b), y contra otros algoritmos basados en sensado compresivo propuestos por Tian (2008), Haque et al. (2015), W. Sun et al. (2015) y Y. Wang et al. (2015); en la figura se puede apreciar que el desempeño de los algoritmos propuestos por Olabiyi y Annamalai (2012a, 2012b), Sun et al. (2015), Tian (2008), Haque et al. (2015) y Wang et al. (2015) es inferior al alcanzado por el algoritmo propuesto, en función de la probabilidad de detección, de la misma forma se aprecia la probabilidad de detección para el algoritmo propuesto es aproximadamente igual a 1 para valores de SNR superiores a $0 \mathrm{~dB}$, obteniendo solo mejores desempeños los algoritmos propuestos por Pourgharehkhan et al. (2015) y Li et al. (2015) los cuales realizan sensado de espectro de banda angosta en sistemas de múltiples antenas que operan a tasas de muestreo iguales o superiores a la tasa de Nyquist. 
Tabla 1: Parámetros de Simulación.

\begin{tabular}{|c|c|}
\hline Parámetro & Valor \\
\hline \multicolumn{2}{|l|}{ Generación Señal Multibanda } \\
\hline Ancho de Banda Señal Multibanda, $B W$ & $20 \mathrm{MHz}$ \\
\hline Ancho de Banda por Canal, $B$ & 3.3MHz \\
\hline Modulación Señal en Canal & 16QAM \\
\hline Tasa de Símbolos, $R s$ & $2 \mathrm{Msps}$ \\
\hline Factor de Roll Off Filtro Transmisión, $\rho$ & 0.5 \\
\hline Tasa Muestreo Nyquist, $f s$ & $40 \mathrm{MHz}$ \\
\hline $\begin{array}{l}\text { Número Muestras Señal Multibanda } \\
\text { (Nyquist), } n\end{array}$ & 2000 \\
\hline Frecuencias de Portadora, $f c$ & $\begin{array}{l}3 \mathrm{MHz}, \\
6 \mathrm{MHz}, \\
9 \mathrm{MHz}, \\
12 \mathrm{MHz}, \\
15 \mathrm{MHz} \text { y } \\
18 \mathrm{MHz}\end{array}$ \\
\hline \multicolumn{2}{|l|}{ Muestreador Sub_Nyquist } \\
\hline Número Muestras Señal Entrada, $n$ & 2000 \\
\hline Número Muestras Señal Salida, $m$ & 100 \\
\hline \multicolumn{2}{|l|}{ Algoritmo de Sensado MAWBCS } \\
\hline Número de Iteraciones (Antenas) & $q$ \\
\hline
\end{tabular}

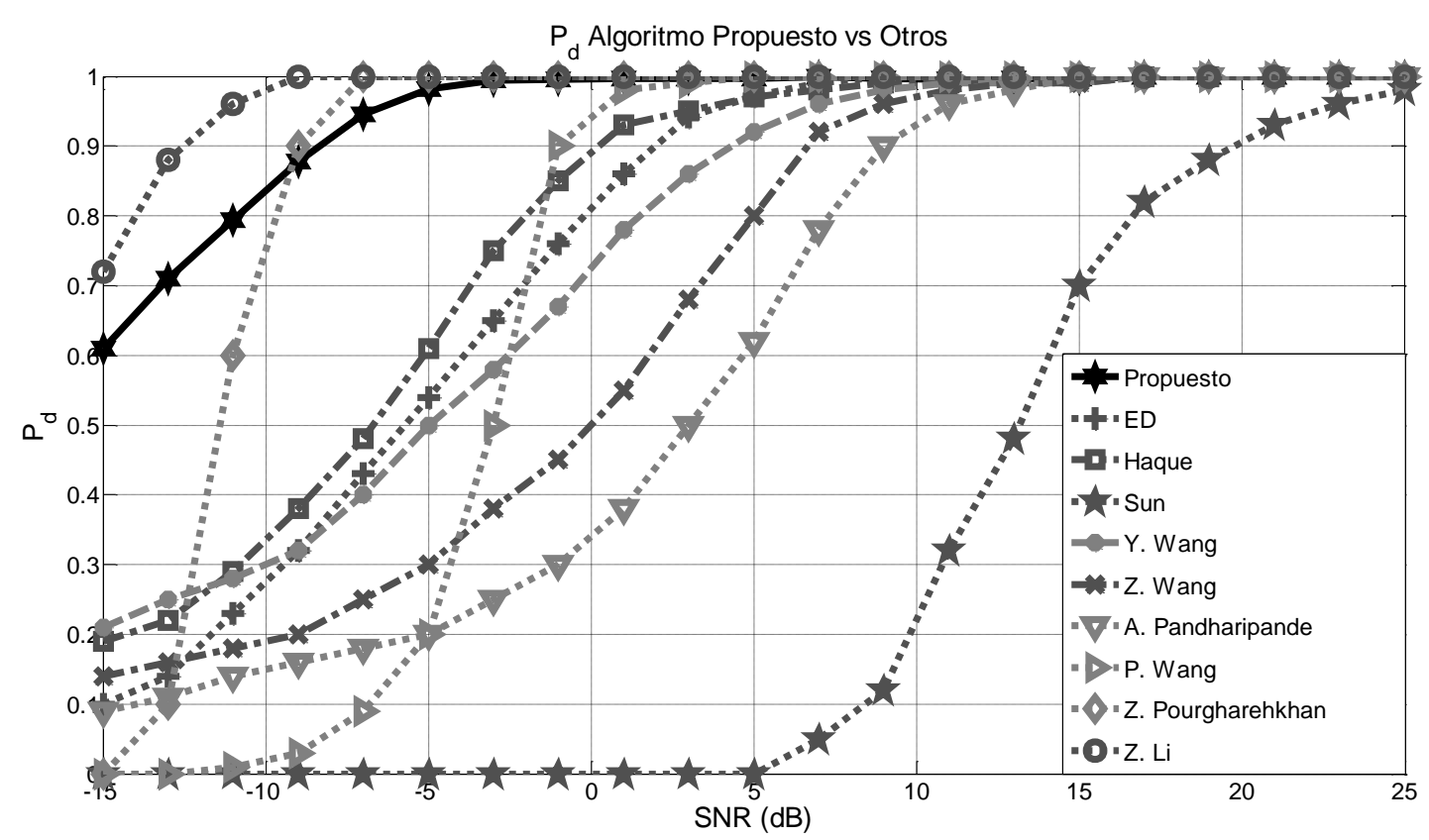

Fig. 5: Desempeño Algoritmo WideBand Compressive Sensing Propuesto vs Algoritmo de Detección de Energía Secuencial Nyquist vs Otros Algoritmos Basados en CS en Función de SNR (2 Antenas).

En la Fig. 6 puede observarse que el mejor desempeño en términos de las curvas de ROC es el correspondiente al algoritmo propuesto, esto debido a que el área bajo la curva del algoritmo propuesto es la mayor, lo cual indica la capacidad del algoritmo de propuesto de identificar acertadamente los WS. Como se aprecia de igual manera en la Fig. 6, el algoritmo con peor desempeño es el propuesto por Sun (Tian, 2008), ya que la curva ROC indica una probabilidad de 0.5 de realizar una detección correcta de los WS. Considerando que los resultados ilustrados en la Fig. 6 corresponden a las curvas ROC de los algoritmos contrastados a una SNR de -3dB, se evidencia de nuevo que el algoritmo propuesto mejora significativamente el desempeño de los otros algoritmos en condiciones de baja SNR, exceptuando solamente el propuesto en (Li et al., 2015), que presenta la desventaja del sensado de banda estrecha a tasas de muestreo iguales o superiores a la tasa de Nyquist. 


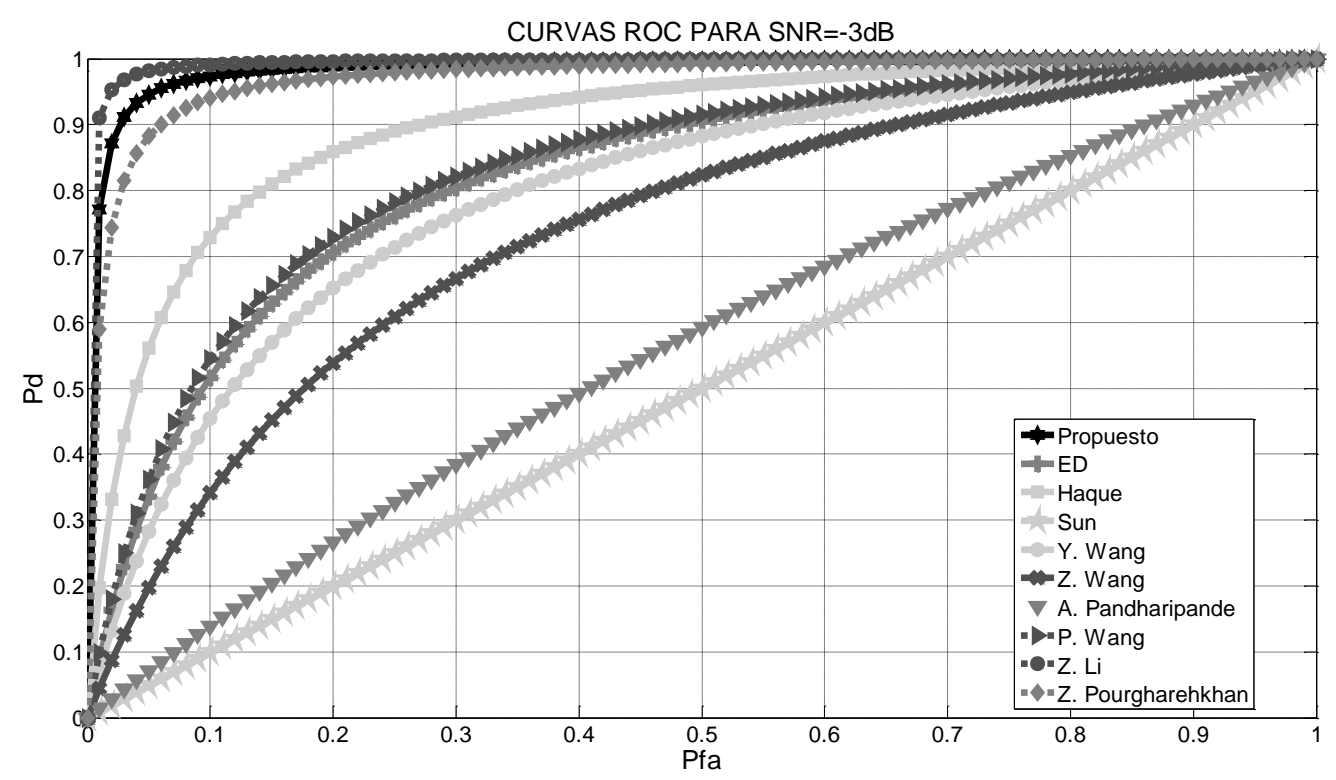

Fig. 6: Curvas ROC para SNR $=-3 \mathrm{~dB}$ (2 Antenas).

En la fig. 7 se ilustra el desempeño del algoritmo propuesto en función de la probabilidad de detección contra la SNR de acuerdo al número de antenas en el CRD. Aquí puede apreciarse que en la medida que el CRD presenta mayor cantidad de antenas receptoras, la SNR a la cual se alcanza una probabilidad de detección aproximadamente igual a uno disminuye, alcanzándose el desempeño objetivo en CR para el estándar 802.22 con una cantidad aproximada de 20 antenas. Así mismo se observa que se mejora el desempeño cuando el CDR incorpora la tecnología MIMO Masivo propuesta para los sistemas móviles e inalámbricos de próxima generación (50 o más antenas).

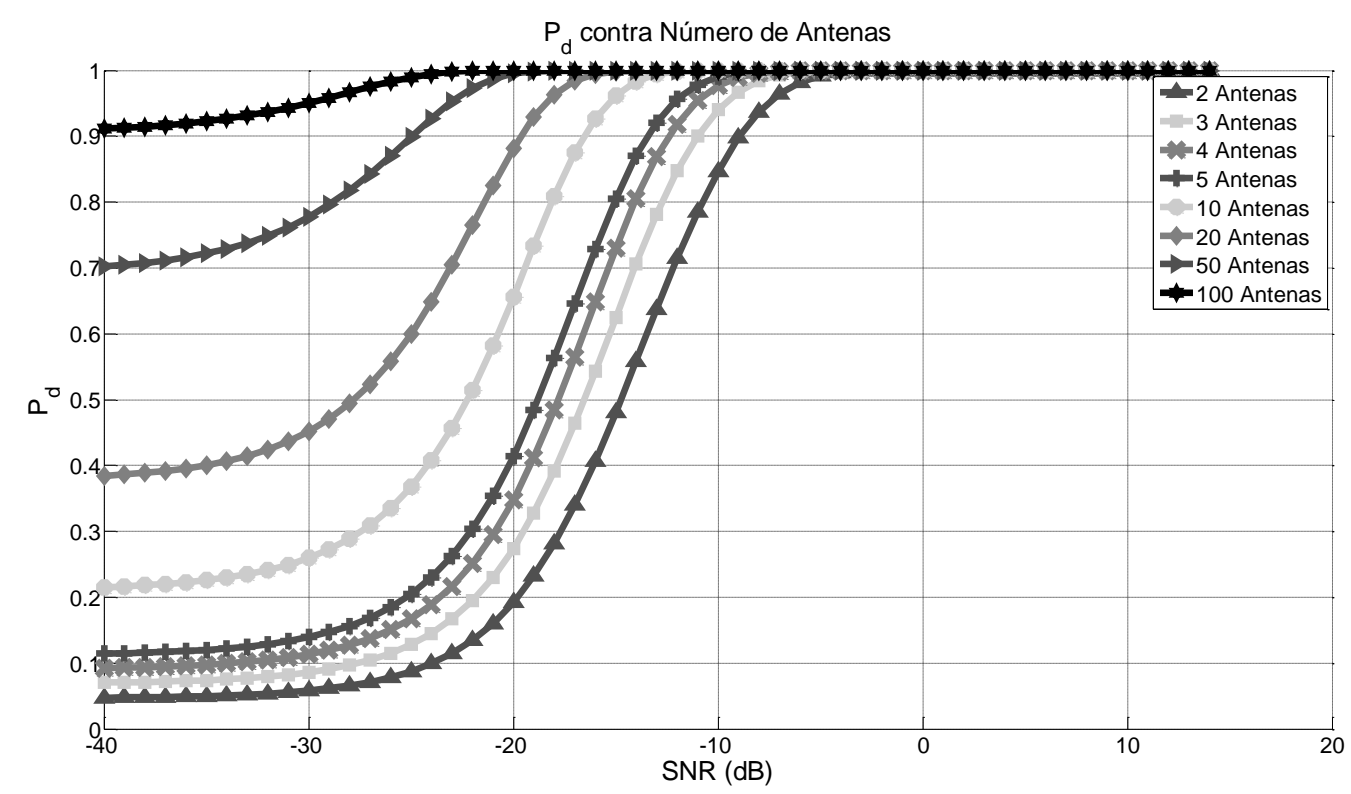

Fig. 7: Probabilidad de Detección Contra SNR en Función del Número de Antenas del CDR.

\section{Análisis de Complejidad Computacional}

La complejidad computacional del algoritmo propuesto puede analizarse en dos etapas. La primera etapa corresponde al sub-muestreo realizado la matriz de muestreo propuesta, en el cual se requieren una cantidad de $\mathrm{Km}$ multiplicaciones escalares presentando una complejidad $O(m)$. La segunda etapa es la correspondiente a la función de sensado, en el cual, como se ilustra en la Fig. 5, la operación de la estimación de la señal se realiza mediante el algoritmo $S V T$, que presenta complejidad $O(n)$ (Cai et al., 2010), posteriormente las líneas 11 a 24 de la Fig. 5 tienen complejidad $3 k+n+5$ y dado que $n>>k$ puede definirse la complejidad como $O(n)$, con lo cual, se define como complejidad de la segunda etapa $O(n)$. En síntesis, la complejidad computacional de las dos etapas que conforman el método propuesto, será la máxima complejidad entre ellas, por lo tanto será $O(n)=$ máx $[O(m), O(n)]$, ya que $m<n$. 


\section{CONCLUSIONES}

En este artículo se propone un novedoso algoritmo local de sensado de espectro de banda ancha en CR multiantena basado en muestreo sub-nyquist, el cual no requiere conocimiento apriori de las características de la señal presente en el entorno de radio, demostrándose que mediante el algoritmo propuesto es posible realizar la función de sensado de espectro de banda ancha utilizando una cantidad de muestras menor a las obtenidas a la tasa de Nyquist, alcanzando un desempeño superior a otros algoritmos propuestos donde se contempla el sensado de espectro con dispositivos secundarios multiantena, al detector de energía secuencial de banda ancha y a los algoritmos de sensado de espectro de banda ancha basados en sensado compresivo evaluados, en régimen de baja SNR.

\section{REFERENCIAS}

Baraniuk, R. G., Compressive sensing, IEEE signal processing magazine, 24(4), 118-121 (2007)

Cai, J.-F., Candès, E. J., y Shen, Z., Singular Value Thresholding Algorithm for Matrix Completion, doi: 10.1137/080738970, SIAM Journal on Optimization, 20(4), 1956-1982 (2010)

Candes, E. J., Romberg, J., y Tao, T., Robust uncertainty principles: exact signal reconstruction from highly incomplete frequency information, doi: 10.1109/TIT.2005.862083, IEEE Transactions on Information Theory, 52(2), 489-509 (2006)

Gnedenko, B. V., y Kolmogorov, A.N., Limit Distributions for Sums of Independent Random Variables, Reading, Mass, Addison-Wesley (1954)

Goldsmith, A., Wireless Communications, Cambridge University Press (2005)

Haque, T., Yazicigil, R. T., Pan, K. J.-L., Wright, J. y Kinget, P. R., Theory and Design of a Quadrature Analogto-Information Converter for Energy-Efficient Wideband Spectrum Sensing, doi: 10.1109/TCSI.2014.2360756, IEEE Transactions on Circuits and Systems I, Regular Papers, 62(2), 527-535 (2015)

Astaiza, E., Jojoa, P. E., y Bermúdez, H. F., Compressive sensing: A methodological approach to an efficient signal processing, doi: 10.15446/dyna.v82n192.45512, DYNA, 82(192), 203-210 (2015)

Laska, J. N., Kirolos, S., Duarte, M. F., Ragheb, T. S., Baraniuk, R. G., y Massoud, Y., Theory and Implementation of an Analog-to-Information Converter using Random Demodulation, doi: 10.1109/ISCAS.2007.378360, in 2007 IEEE International Symposium on Circuits and Systems, IEEE 19591962 (2007)

Li, Z., Wang, D., Qi, P., y Hao, B., Maximum Eigenvalue Based Sensing and Power Recognition for MultiAntenna Cognitive Radio System, doi: 10.1109/TVT.2015.2511783 IEEE Transactions on Vehicular Technology, PP(99), 1-11 (2015)

Mishali, M., y Eldar, Y. C., Blind Multiband Signal Reconstruction: Compressed Sensing for Analog Signals, doi: 10.1109/TSP.2009.2012791, IEEE Transactions on Signal Processing, 57(3), 993-1009 (2009)

Mishali, M., Eldar, Y. C., Dounaevsky, O., y Shoshan, E., Xampling: Analog to digital at sub-Nyquist rates, doi: 10.1049/iet-cds.2010.0147, IET Circuits, Devices \& Amp, Systems, 5(1), 8-20 (2011)

Mitola III, J., Cognitive Radio An Integrated Agent Architecture for Software Defined Radio. Royal Institute of Technology (KTH), Sweden (2000)

Natarajan, B. K., Sparse Approximate Solutions to Linear Systems, doi: 10.1137/S0097539792240406, SIAM Journal on Computing, 24(2), 227-234 (2006)

Olabiyi, O., y Annamalai, A., Extending the capability of energy detector for sensing of heterogeneous wideband spectrum, doi: 10.1109/CCNC.2012.6181033, in 2012 IEEE Consumer Communications and Networking Conference (CCNC), IEEE 454-458 (2012a)

Olabiyi, O., y Annamalai, A., Parallel multi-channel detection: A practical solution to energy detection of heterogeneous wideband spectrum, doi: 10.1109/SARNOF.2012.6222719, in Sarnoff Symposium (SARNOFF), 35 th $I E E E, 1-5(2012 b)$ 
Pandharipande, A., y Linnartz, J.-P. M. G., Performance Analysis of Primary User Detection in a Multiple Antenna Cognitive Radio, doi: DOI:10.1109/ICC.2007.1072, in 2007 IEEE International Conference on Communications, IEEE 6482-6486 (2007)

Pourgharehkhan, Z., Taherpour, A., Sala-Alvarez, J., y Khattab, T., Correlated Multiple Antennas Spectrum Sensing Under Calibration Uncertainty, doi: 10.1109/TWC.2015.2459706, IEEE Transactions on Wireless Communications, 14(12), 6777-6791(2015)

Recht, B., A Simpler Approach to Matrix Completion. ACM DL, The Journal of Machine Learning Research, $12,3413-3430$ (2011)

Sun, H., Nallanathan, A., Wang, C.-X., y Chen, Y., Wideband spectrum sensing for cognitive radio networks: a survey, doi: 10.1109/MWC.2013.6507397, IEEE Wireless Communications, 20(2), 74-81 (2013)

Sun, W., Huang, Z., Wang, F., y Wang, X., Compressive wideband spectrum sensing based on single channel, doi: 10.1049/el.2014.4223, Electronics Letters, 51(9), 693-695 (2015)

Tian, Z., Compressed Wideband Sensing in Cooperative Cognitive Radio Networks, doi: 10.1109/GLOCOM.2008.ECP.721, in IEEE GLOBECOM 2008 - 2008 IEEE Global Telecommunications Conference, IEEE 1-5 (2008)

Wang, P., Fang, J., Han, N., y Li, H., Multiantenna-Assisted Spectrum Sensing for Cognitive Radio, doi: 10.1109/TVT.2009.2037912, IEEE Transactions on Vehicular Technology, 59(4), 1791-1800 (2010)

Wang, Y., Guo, C., Sun, X., y Feng, C., Time-Efficient Wideband Spectrum Sensing Based on Compressive Sampling, doi: 10.1109/VTCSpring.2015.7146135, in 2015 IEEE 81 ${ }^{\text {st }}$ Vehicular Technology Conference (VTC Spring), 1-5 (2015)

Yen, C.-P., Tsai, Y., y Wang, X., Wideband Spectrum Sensing Based on Sub-Nyquist Sampling, doi: 10.1109/TSP.2013.2251342, IEEE Transactions on Signal Processing, 61(12), 3028-3040 (2013) 